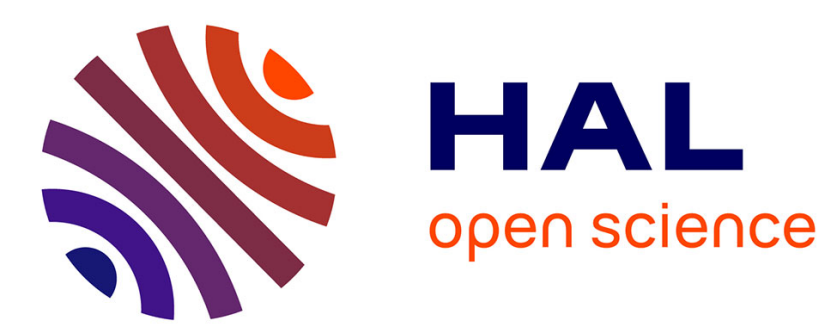

\title{
A theory of thin lamellar eutectic growth with anisotropic interphase boundaries
}

S Akamatsu, S Bottin-Rousseau, M Serefog ${ }^{` l u, ~ G ~ S ~ F a i v r e ~}$

\section{To cite this version:}

S Akamatsu, S Bottin-Rousseau, M Serefog ${ }^{`} l u$, G S Faivre. A theory of thin lamellar eutectic growth with anisotropic interphase boundaries. Acta Materialia, 2012, 60, pp.3199 - 3205. 10.1016/j.actamat.2012.02.031 . hal-01448369

\section{HAL Id: hal-01448369 \\ https://hal.science/hal-01448369}

Submitted on 22 Feb 2017

HAL is a multi-disciplinary open access archive for the deposit and dissemination of scientific research documents, whether they are published or not. The documents may come from teaching and research institutions in France or abroad, or from public or private research centers.
L'archive ouverte pluridisciplinaire HAL, est destinée au dépôt et à la diffusion de documents scientifiques de niveau recherche, publiés ou non, émanant des établissements d'enseignement et de recherche français ou étrangers, des laboratoires publics ou privés. 


\title{
A theory of thin lamellar eutectic growth with anisotropic interphase boundaries
}

\author{
S. Akamatsu*, S. Bottin-Rousseau, M. Şerefoğlu** and G. Faivre \\ Institut des Nanosciences de Paris, UPMC, CNRS, 4 place Jussieu, 75252 Paris Cedex 05, France
}

\begin{abstract}

\section{Rotating-I-esub3}

We present a semiempirical theory of the effects of an orientation dependence of the surface free energy of interphase boundaries (interphase boundary anisotropy) on lamellar eutectic growth in thin samples. We show that, to a good approximation, thin lamellar eutectic patterns with a strong interphase boundary anisotropy travel along the growth front at such a velocity - or, equivalently, at such an inclination angle of the lamellae left behind in the solid - that the surface tension force of the interphase boundary is nearly parallel to the applied thermal gradient. This explains, among other things, the crystallographic locking of lamellar eutectic patterns that occurs in those eutectic grains, which have cusp singularities in the Wulff plot of the interphase boundary. Based on this theory, we show that the rotating directional solidication (RDS) method, by which a thin sample is rotated with respect to a fixed unidirectional thermal gradient, must yield eutectic lamellae whose trajectories are nearly homothetic to the 2D Wulff form of the interphase boundary. This opens up new possibilities for the experimental study of interphase boundary anisotropy in eutectic alloys.
\end{abstract}

Key words: Eutectic, directional solidification, microstructures, interphase boundaries

*Corresponding author

** Present address: Department of Mechanical Engineering, Koç University, Rumeli Feneri Yolu, 34450 Sariyer, Istanbul, Turkey

Email address: akamatsu@insp.jussieu.fr (S. Akamatsu ) 


\section{Introduction}

The important effect of an orientation dependence of the surface free energies of interfaces on the morphology of eutectic microstructures has long been recognized. However, our understanding of this effect is still fragmentary. Following Jackson and Hunt's landmark articles of 1966 [1, 2], attention has been mostly focussed on the type (nonfaceted or faceted) of anisotropy exhibited by solid-liquid interfaces. The theory of eutectic solidification in alloys with nonfaceted solidliquid interfaces (nf/nf eutectics) was developed under the assumption of a complete isotropy of all interfaces. The famous regularity-i.e. space periodicity and steadiness-of lamellar eutectic growth patterns was established theoretically under this assumption. The full success of this "isotropic" theory in suitably chosen eutectic grains $[3,4,5]$ should not eclipse the importance of crystallographic effects linked to interphase boundary anisotropy in other eutectic grains. Numerous metallic eutectics, notably $\mathrm{Al}-\mathrm{Al}_{2} \mathrm{Cu}$ [6] or $\mathrm{Al}-\mathrm{Al}_{2} \mathrm{Cu}$-based ternary eutectics [7], form "epitaxial" eutectic grains (i.e. eutectic grains with orientation relationships between phases such that there exists low-energy orientations for the interphase boundary) upon directional solidification [8]. In epitaxial eutectic grains, lamellar planes are locked onto low-energy orientations of the interface boundary and are therefore generally steeply inclined with respect to the direction of growth, in contradiction with the isotropic theory. These well-known facts pose two as yet unsolved problems. First, what is the selection mechanism of the epitaxial eutectic grains during directional solidification? Second, what is the locking mechanism of eutectic lamellae in epitaxial eutectic grains? This study is dealing with the latter question and focuses on two-dimensional (thin) eutectics. The phenomenon of crystallographic locking of the lamellar plane has previously been observed in thin samples of carbon tetrabromide-hexachloroethane $\left(\mathrm{CBr}_{4}-\mathrm{C}_{2} \mathrm{Cl}_{6}\right)[2,9,10]$, which is a transparent analog of $\mathrm{nf} / \mathrm{nf}$ metallic eutectics, and $\mathrm{In}-\mathrm{In}_{2} \mathrm{Bi}$ [11]. Interestingly, crystallographic locking has been sought but could not be found in the transparent nf/nf succinonitrile-(D)camphor (SCN-DC) eutectic, probably because no epitaxial interphase orientation relationships exist in this alloy [12].

The explanations of crystallographic locking that have previously been proposed were unsatisfactory because they did not take into account the dynamical (i.e. out-of-equilibrium) nature of lamellar eutectic patterns [8]. In this article, we propose a dynamical theory of crystallographic locking, and, more generally, of interphase boundary anisotropy effects in lamellar eutectic growth. It is known that the steady-state ( $s t$ ) lamellar patterns of eutectics with some anisotropy of any (solid-liquid or interphase) interface must travel along the growth front at a con- 
stant velocity $v_{s t}[10,13]$. These "anisotropy-driven travelling " patterns leave behind in the solid interphase boundaries that are inclined with respect to the growth axis $\mathbf{z}$ at an angle $\phi_{s t}=-\arctan \left(v_{s t} / V\right)$, where $V$ is the growth velocity. This in turn modifies the equilibrium between surface tension forces at the junctions between the three phases on the growth front, and thus the shape of the solid-liquid interfaces. In this article, we focus on systems where the anisotropy of the solidliquid interfaces is negligible compared to that of the interphase boundaries. Our central assertion is that, in such systems, the shape of the solid-liquid interfaces in the anisotropy-driven travelling lamellar patterns can be assumed to be mirror symmetric. In other words, $\phi_{s t}$ is such that the surface tension force $\sigma$ of the interphase boundary is approximately aligned with $\mathbf{z}$ (Fig. 1). This assumption will be referred to as the "symmetric-pattern" or " $\sigma \| \mathbf{z}$ " approximation thereafter. We first present the experimental and theoretical arguments that support this assumption. Next we show that crystallographic locking can be coherently explained on the basis of this assumption. Finally, we study the trajectories followed by eutectic lamellae during a rotating directional solidification (RDS) experiment, i.e., when a thin sample is rotated with respect to a fixed unidirectional thermal gradient, and show that, in the symmetric-pattern approximation, these trajectories are homothetic to the 2D Wulff form of the interphase boundary in the sample plane. Experimental observations made by the RDS method will be presented in a forthcoming article.

\section{Basic concepts}

To avoid any obscurity, a few basic concepts should first of all be recalled. A eutectic grain (EG) is a portion of the solid, inside which the crystal-lattice orientation of either eutectic phase is uniform [8]. Morphology apart, it is a heterophase bicrystal defined by two sets of orientational variables, one describing the interphase orientation relationship and the other the orientation of the whole EG with respect to a frame of reference attached to the experimental setup, e.g. the $\mathbf{x y z}$ reference frame defined in Fig. 1 [14]. Let $\hat{\boldsymbol{n}}$ be the unit normal vector and $\gamma(\hat{\boldsymbol{n}})$ the surface free energy of the interphase boundary in a given EG. The 3D Wulff plot of the interphase boundary in this EG is the surface described by $\boldsymbol{r}=\boldsymbol{\gamma}$, where $\boldsymbol{r}$ is the position vector and $\boldsymbol{\gamma}=\gamma(\hat{\boldsymbol{n}}) \hat{\boldsymbol{n}}$. The corresponding Wulff form (form of minimum surface energy for a given volume) is described by $\boldsymbol{r}=\boldsymbol{\xi}_{3 \boldsymbol{D}}$, where $\boldsymbol{\xi}_{3 \boldsymbol{D}}=\boldsymbol{\gamma}+|\boldsymbol{r}| \nabla_{a} \gamma, \nabla_{a}$ representing the angular part of the gradient operator [15]. Let $\hat{l}$ be the unit tangent to a trijunction line between the interphase boundary and the solid-liquid interfaces. The equation for the equilibrium between surface 
tensions (Young-Herring's equation) at the trijunctions reads

$$
\gamma_{a}+\gamma_{b}+\sigma=\mathbf{0},
$$

where $\sigma=\xi_{3 D} \times \hat{l}$, while $\gamma_{a}$ and $\gamma_{b}$ are the surface tension forces of the isotropic solid-liquid interfaces.

Let us now turn to thin-sample directional solidification. By "thin", it is meant that the confinement of the system by the sample walls imposes a pronounced 2D character to the solidification dynamics, so that the interfaces are constrained to remain essentially perpendicular to the sample plane [16]. Exceptions to this rule called "out-of-plane lamellar patterns" will be considered later on in this article. For now, we assume a strict bidimensionality, i.e. $\hat{\boldsymbol{n}}$ is parallel to the sample plane, so that $\gamma$ depends on a single variable, e.g. the inclination angle $\phi$ of the interphase boundary. The 2D Wulff plot of this boundary is the cross section of its 3D Wulff plot by the $\mathbf{x z}$ plane, while its 2D Wulff form is described by $\boldsymbol{r}=\boldsymbol{\xi}$, where $\boldsymbol{\xi}$ is the orthogonal projection of $\boldsymbol{\xi}_{3 D}$ onto the $\mathbf{x z}$ plane. One has $\boldsymbol{\xi}=\gamma \hat{\boldsymbol{n}}-\gamma^{\prime} \hat{\boldsymbol{t}}$ and $\boldsymbol{\sigma}=\gamma \hat{\boldsymbol{t}}+\gamma^{\prime} \hat{\boldsymbol{n}}$, where $\gamma^{\prime}=\mathrm{d} \gamma / \mathrm{d} \phi$ and $\hat{\boldsymbol{t}}=-d \hat{\boldsymbol{n}} / d \phi$ (Fig. 2). Introducing the angular deviation $\psi=\arctan \left(\gamma^{\prime} / \gamma\right)$ of $\sigma$ from the interface boundary, and the inclination angle $\delta$ of $\sigma$, we write the $\sigma \| \mathbf{z}$ condition in the form of an equation for $\phi$,

$$
\delta(\phi)=\phi+\psi(\phi)=0 .
$$

The solutions to Eq. 2 may be formally written

$$
\phi_{s t}=\delta^{-1}(0),
$$

provided it is accepted that $\delta^{-1}$ can be a multivalued function. In fact, an elementary calculation leads to $d \delta / d \phi=\gamma\left(\gamma+\gamma^{\prime \prime}\right) /\left(\gamma^{2}+\gamma^{\prime 2}\right)$. Thus $\delta^{-1}$ is single-valued when the surface stiffness $\tau=\gamma+\gamma^{\prime \prime}$ of the interphase boundary is positive at all $\phi$. In this case, $\phi_{s t}$ is unique. Conversely, $\delta^{-1}$ is multivalued when the interface boundary has one, or several, orientation ranges in which $\tau$ is negative, in which case $\phi_{s t}$ may not be unique. It should be noted that $\tau(\phi)>0$ is the Herring condition for an interface to be stable against a hill-and-valley instability [17]. This issue is further discussed below. We conclude this introductory section by noting that $\delta$ is the polar angle of $\xi$ and that $d \xi / d \phi=\gamma^{\prime}\left(\gamma+\gamma^{\prime \prime}\right) / \sqrt{\gamma^{2}+\gamma^{\prime 2}}$, where $\xi=|\xi|$. One therefore has

$$
\frac{d \xi}{\xi d \delta}=\frac{d \gamma}{\gamma d \phi},
$$

which is the differential equation for the 2D Wulff form of the interphase boundary in polar coordinates, assuming $\gamma(\phi)$ and hence $\phi(\delta)$ to be known. 


\section{The symmetric-pattern approximation}

The symmetric-pattern assumption was primarily made on the basis of experimental observations that showed space periodic lamellar patterns travelling laterally at relatively large velocities and nevertheless exhibiting nearly mirror symmetric shapes of their growth fronts (see Section 5). This feature is in strong contrast with the spectacularly asymmetric growth front shapes of the so-called "tilted" lamellar patterns that are observed in off-eutectic isotropic systems at large values of the interlamellar spacing $\lambda$-specifically, above a threshold spacing value $\lambda_{\text {tilt }} \approx 1.5 \lambda_{J H}$, where $\lambda_{J H}$ is the Jackson-Hunt $(\mathrm{JH})$ minimum-undercooling spacing $[3,4]$. A comparison between the growth-front shapes of the two types of travelling patterns clearly shows that, in a mirror symmetric travelling pattern, $\sigma$ must be parallel to $\mathbf{z}$, and not to the interphase boundaries, and that, therefore, the interphase boundaries must have a strong (i.e. such that $\gamma^{\prime} / \gamma \sim 1$ ) capillary anisotropy (Fig. 3).

An analytical demonstration of the validity of the symmetric-pattern assumption should proceed as follows. The equations to be considered are those of the JH model of 2D eutectic directional solidification [2], which include a diffusion equation for the solute in the liquid and, at the solid-liquid interface, a mass conservation equation and two local-equilibrium (Gibbs-Thomson and Young-Herring) equations. As is well known, Jackson and Hunt solved these equations using a planar-front approximation in the $\lambda V / D<<1$ limit, where $\mathrm{D}$ is the solute diffusion coefficient in the liquid, and found a continuous set of steady, space periodic, mirror-symmetric solutions over a wide $\lambda$-range at given $V$. The fact that the solutions have mirror symmetry follows from the invariance of the governing equations with respect to mirror reflexion about yz. This invariance is destroyed, in general, by the introduction of interfacial anisotropy in the local-equilibrium equations. Consequently, the space periodic solutions of the $\mathrm{JH}$ model with anisotropic interfaces do not have mirror symmetry and travel along $\mathbf{x}$ at a finite velocity $v_{s t}$. In other words, they are steady in a frame of reference travelling at $v_{s t}$ along $\mathbf{x}$. The differences in concentration field and form of the solid-liquid interface between anisotropic patterns and isotropic ones arise from the additional "lateraladvection" terms appearing in the diffusion and mass conservation equations when moving from a fixed to a travelling reference frame. Reproducing JH's calculation while taking the lateral-advection terms into consideration leads to the conclusion that the asymmetric components of the concentration field and of the form of the solid-liquid interface due to lateral advection are little-o of $\lambda V / D$, and are therefore negligible. This, strictly speaking, does not mean that the symmetric-pattern 
approximation is accurate when $\lambda V / D<<1$, but only that its accuracy cannot be evaluated using a planar-front approximation. However, it strongly suggests that the accuracy of the symmetric-pattern approximation increases as the contact angles (angles with respect to $\mathbf{x}$ at the trijunctions) of the solid-liquid interfaces decrease, everything else equal, and that the approximation becomes exact in the limit of vanishing contact angles. To sum up, numerical simulations will be necessary to evaluate the accuracy of the symmetric-pattern approximation as a function of, in particular, the contact angles of the solid-liquid interfaces. For now, based on experimental observations, the symmetric-pattern approximation can be safely assumed to be at least semiquantitatively valid for the determination of the anisotropy-driven travelling velocities of lamellar patterns.

\section{Locked versus floating lamellar patterns}

Let us consider a EG, in which the 2D Wulff plot of the interphase boundary has a trough with a pointed end (cusp) at $\phi=\theta$, where $\theta$ is the inclination angle of the corresponding facet in the Wulff form. It is assumed that this facet has a high mobility so that Young-Herring's equilibrium condition can be fulfilled at, and near, $\phi=\theta$. Equivalently, it may be assumed that the pointed end of the trough is in fact rounded within a very small angular range [18, 19]. Anticipating on Section 6, we furthermore allow the EG to be rotated about $\mathbf{y}$. Then $\theta$ represents the rotation angle of the EG measured from the position at which the facet is parallel to $\mathbf{z}$. We may write $\gamma(\phi, \theta)=\gamma_{0}(\phi-\theta)$, where $\gamma_{0}(\phi)$ is the Wulff plot of the interphase boundary at $\theta=0$ and reformulate Eq. 3 as

$$
\phi_{s t}(\theta)=\delta_{0}^{-1}(-\theta)+\theta,
$$

where $\delta_{0}^{-1}$ is the inverse function of $\delta_{0}(\phi)=\phi+\arctan \left(\gamma_{0}^{\prime} / \gamma_{0}\right)$. For ease of reference, the discussion of Eq. 5 will use an elementary model of a Wulff plot with a pointed trough. In suitable surface energy units, the model is

$$
\gamma_{0}(\phi)=1-\epsilon \frac{1-\cos (|\phi|-\Phi)}{1-\cos \Phi}
$$

for $|\phi| \leq \Phi$, and $\gamma_{0}(\phi)=1$ for $|\phi| \geq \Phi$. In this model, $\epsilon$ represents the relative depth of the trough and $\Phi$ its angular width. For illustration purposes, we can set $\Phi$ at some typical value for heterophase interfaces, e.g. $0.2 \mathrm{rad}$ [14], and keep only $\epsilon$ as a parameter. The surface stiffness is constant and equal to $1-\epsilon / \epsilon_{s}$, where $\epsilon_{s}=1-\cos \Phi$, inside the trough, and is equal to unity ouside the trough. 
Thus the interphase boundary is stable everywhere when $\epsilon<\epsilon_{s}$ and unstable in the orientation ranges corresponding to the flanks of the trough ("vicinal" ranges) when $\epsilon>\epsilon_{s}$. Figure 4 shows the aspect of three key functions [the Wulff plot, the Wulff form, the $\phi_{s t}(\theta)$ function] in this model at $\epsilon<\epsilon_{s}$. The discontinuity of $\gamma_{0}^{\prime}$ occurring at the bottom of the trough gives rise to a facet in the Wulff form, as is well known. In the $\phi_{s t}(\theta)$ curve, it gives rise to a line segment $\left(c^{\prime} c\right)$ of slope unity. The interphase boundary is parallel to the facet for all $\theta$ belonging to the finite-width range (the "locking range") spanned by this segment. This is the phenomenon known as crystallographic locking.

In Fig. 4, the transition from the locked $\left(c^{\prime} c\right)$ to a non-locked branch of states (e.g. $p q$ ) as a function of $\theta$ occurs continuously through a branch of stable intermediate states $(c p)$. However, the measured values of $\epsilon$ reported for low-energy interphase interfaces [14] are generally above the instability threshold value $\left(\epsilon_{s} \approx 0.02\right)$ according to the above model. Situations where $\epsilon>\epsilon_{s}$ must therefore be frequently observed. In this case, Figure 5 shows that two features arise: the locked branch overlaps the non-locked ones; the intermediate branches are unstable with respect to Herring's instability. Therefore, locked and non-locked lamellar patterns are metastable with respect to each other within a certain orientation range. Hysteresis and spatial coexistence of different lamellar patterns can in principle be observed. The transitions between the two types of patterns as a function of $\theta$ must be discontinuous.

Qualitatively speaking, the above considerations are independent of the model chosen. Therefore, two conclusions of general validity may be drawn: first, crystallographic locking is due to the existence of cusp-like singularities at the minima of the Wulff plot of the interphase boundary, and is therefore specific for epitaxial EGs; second, discontinuous transitions between locked and non-locked lamellar patterns should be observed when, and only when, the vicinal orientations of the cusps are unstable with respect to Herring's instability. These conclusions emphasize the similarity that exists between crystallographic locking of lamellar patterns and faceting of equilibrium forms. There is however a significant difference between the two phenomena, which we will now stress. As is well known, isothermal equilibrium shapes must be convex, and therefore cannot coincide with theoretical Wulff forms in, and around, unstable orientation ranges. The orientation ranges eliminated from ("forbidden" in) the equilibrium forms include not only unstable segments but also adjacent stable segments, such as segments ec and ep in Fig. 5a. No similar elimination needs to occur in lamellar eutectic growth. As far as we can see, $\phi_{s t}$ can take any value belonging to a stable branch of the $\phi_{s t}(\theta)$ diagram. Hence possible hysteresis and coexistence effects, as mentioned above. 
Finally, a remark about the dynamics of locked lamellar patterns should be made. Experimental observations, which will be reported in a future article, showed locked lamellar patterns with very irregular $\lambda(x)$ distributions (i.e. spacing distributions along the growth front) that did not evolve over time. This is in strong contrast with isotropic lamellar patterns, which, as is well known, are steady only when their spacing distribution is uniform. More specifically, an isotropic lamellar pattern with a spatial modulation in $\lambda$ relaxes toward uniformity through a process called phase-diffusion (or $\lambda$-diffusion) whose time constant varies as the square of the wavelength of the modulation [20,21]. This is a linear-response mechanism, which relies on the fact that all the features of the pattern, especially, the shape of the solid-liquid interface, vary smoothly with $\phi$. It should be clear from the foregoing that, in locked lamellar patterns, $\sigma$, and hence the shape of the solid-liquid interface, vary discontinuously at $\phi=\theta$. Therefore, locked lamellar patterns - at least, those with hysteretic transitions between locked and non-locked patternsare deprived of a linear mechanism of response to spatial variations of $\lambda$, in agreement with experimental observations. This leads us to an important terminological remark. We propose to define locked lamellar patterns as those patterns, in which the $\lambda$-diffusion mechanism is blocked. Correlatively, those lamellar patterns, be they isotropic or anisotropic, in which $\lambda$-diffusion is effective should be grouped into one category, which, following Ref [10], we propose to call "floating" .

\section{Out-of-plane locked lamellar patterns}

Figure 6 displays high-magnification views of three different types of lamellar patterns observed in different EGs during standard (as opposed to rotating) directional solidification in a $12 \mu$ m-thick sample of $\mathrm{CBr}_{4}-\mathrm{C}_{2} \mathrm{Cl}_{6}$ [4]. The facts to note here are (i) the mirror symmetry (within experimental error) of the form of the growth fronts in travelling lamellar patterns, (ii) the low value of the contact angles in locked patterns compared to isotropic ones, and (iii) the breaking of the bidimensionality condition in the "out-of-plane" locked pattern of Fig. 6c. The first fact is key for the validity of the symmetric-pattern approach, as was stressed above. The second one is a confirmation that locked lamellar planes correspond to low-energy interphase boundaries. The third fact will now be commented.

Out-of-plane locked patterns break the bidimensionality condition in spite of strong confinement effects. Clear examples of such patterns were identified in-situ through their optical contrast in $\mathrm{CBr}_{4}-\mathrm{C}_{2} \mathrm{Cl}_{6}$, as illustrated in Fig. 6c, and through post-mortem transverse metallographic sections in $\mathrm{In}_{-} \mathrm{In}_{2} \mathrm{Bi}$ [11]. These patterns had irregular $\lambda(x)$ distributions indicating that they were locked onto facet planes 
corresponding to out-of-plane cusps of the 3D Wulff plot of the interphase boundary. Given that reflection boundary conditions are imposed on the concentration field at the sample walls, the question arises as to the geometry of out-of-plane locked patterns near these walls. It should be noted that the 3D extension of an out-of-plane locked pattern by symmetry about the sample walls is a chevron pattern, which suggests the presence of a localized defect line along the walls [22]. For our present purpose, however, the most important point is as follows. A thin eutectic system that displays an out-of-plane locked pattern also possesses, as a general rule, a 2D solution, either locked of floating, to the equations of growth. There may be conditions under which these two patterns are metastable with respect to each other.

\section{Rotating directional solidification}

A RDS experiment is schematically represented in Figure 7a. A thin sample is placed in a directional thermal gradient, and then rotated at an angular speed $\omega=d \theta / d t$ about the $\mathbf{y}$ axis. A $2 \mathrm{D}$ reference frame $\mathbf{X Z}$ with the same origin $\Omega$ as the laboratory reference frame is attached to the rotating sample. We focus on the growing side $(x>0)$ of the solidification front. We neglect the undercooling of the growth front, which is thus equated with the isotherm at $T_{E}$. We assume the sample to be a single eutectic grain and $\Omega$ to sit at a fixed distance $R$ from the $T_{E^{-}}$ isotherm. We seek a differential equation for RDS lamellar trajectories under the $\boldsymbol{\sigma} \| \mathbf{z}$ assumption. More specifically, we assume that RDS lamellar trajectories adjust their inclination angle to the solution of Eq. 2 instantaneously. We thus neglect any effect that may arise from a non-uniformity of $\lambda(x)$ or from the spatial $V$-ramp generated by the RDS. Let $\mathbf{M}$ be the position vector of a trijunction at a given time and $\hat{\boldsymbol{x}}$ and $\hat{z}$ be the unit translation vectors of the $\mathbf{x}$ and $\mathbf{z}$ axis (Fig. 7b). We set $\mathbf{M}=\rho(\theta) \hat{\boldsymbol{x}}+R \hat{\boldsymbol{z}}$, where $\rho(\theta)$ is the unknown. An elementary calculation leads to: $d \mathbf{M} / d t=v_{s t} \hat{\boldsymbol{x}}+V \hat{z}$, where $V=\omega \rho$ and $v_{s t}=\omega(d \rho / d t-R)$, and hence

to: $\tan \phi=-(\mathrm{d} \rho / \mathrm{d} \theta-R) \rho$. Substituting $-\psi$ for $\phi$ in accordance with the $\sigma \| \mathbf{z}$ assumption, one obtains:

$$
\frac{d \rho}{d \theta}-\tan [\psi(\theta)] \rho=R
$$

When $R=0$, Eq. 7 is identical to Eq. 4. Thus RDS lamellar trajectories are homothetic to the Wulff form of the interphase boundary. This conclusion is unambiguous when there is no forbidden orientation range in the Wulff plot of the 
interphase boundary. When such a range exists, all the stable branches of the theoretical Wulff form, including those that may be "forbidden" from the equilibrium shape, must be taken into consideration, as explained in Section 4. The theory presented here does not specify the RDS lamellar trajectories in the forbidden ranges. It only indicates that these trajectories are likely to present serial sharp edges, i.e. zig-zag shapes, due to the hysteretic nature of the transition between locked and floating lamellar patterns in theses ranges.

We now assume that $R \neq 0$, as generally occurs in the experiments. Then the solutions to Eq. 7 are in the form

$$
\rho=\rho_{0} F(\theta)+R S(\theta)
$$

where $\rho_{0}=\rho(\theta=0)$,

$$
F(\theta)=\exp \left[\int_{0}^{\theta} \tan \left[\psi\left(\theta^{\prime}\right)\right] d \theta^{\prime}\right],
$$

and

$$
S(\theta)=F(\theta) \int_{0}^{\theta} 1 / F\left(\theta^{\prime}\right) d \theta^{\prime} .
$$

The function $F(\theta)$, which represents the Wulff form, is $2 \pi$-periodic, but $S(\theta)$ is not. Thus RDS lamellar trajectories are spiraling either toward the center $(R<0)$ or the periphery $(R>0)$ of the sample with a separation distance between successive turnings of about $2 \pi R$. The growth pattern correspondingly undergoes a global lateral drift in the laboratory reference frame, so that the centre of the sample is a lamellar sink and its periphery a lamellar source when $R<0$, and vice versa when $R>0$. It should be stressed that this effect has nothing to do with the dynamics of the growth pattern itself, but arises from the translational motion undergone by the V-ramp when $R \neq 0$ (Fig. 7b). Other remarks that will be useful in discussing the experimental results are:

1- RDS lamellar trajectories do not depend on the relation between $\theta$ and $t$;

2 - The evolution with $\theta$ of an initially uniform spacing distribution is given by $\lambda(\theta) / \lambda(0)=W(\theta)$. Any quasi-steady non-uniform spatial distribution of $\lambda$ will undergo a periodic succession of dilatations and compressions but will not change shape during RDS - assuming that the variations of $\lambda$ during a cycle remain inside the stability interval of the pattern [21];

3 - The value of $R$ can be calculated after the end of the experiments by fitting theoretical RDS lamellar trajectories onto the measured ones. In isotropic systems, RDS lamellar trajectories are involutes of the circle of radius $R$ and centre $\omega$ 
and have an explicit intrinsic equation, namely, $s=R\left(\phi-\phi_{0}\right)^{2} / 2$, where $s$ is the arc length of the trajectory and $\phi_{0}$ is a constant, which facilitates the determination of $R$.

4- The Wulff plot of an interface boundary can be calculated from experimental $\phi(\theta)$ data using Eq. 9 and the symmetric-pattern assumption without knowing $R$.

\section{Conclusion}

We have studied theoretically the steady-state dynamics of traveling lamellareutectic patterns during directional solidification of a thin nonfaceted eutectic alloy with a strong capillary anisotropy of the interphase boundaries. Two main conclusions have been reached. The first one is the validity of the so-called symmetric-pattern approximation, which states that, in a traveling lamellar-eutectic pattern, the shape of the solid-liquid interfaces is nearly mirror symmetric. The second one is the homothety between lamellar trajectories and Wulff forms of interphase boundaries during rotating directional solidification, which illustrates the relevance of that experimental method to semiquantitative studies of the capillary anisotropy of eutectic interphase boundaries. New insights have also been gained about the nature and dynamics of crystallographically locked lamellar patterns. These theoretical results will be compared with RDS experimental observations in a future article.

\section{Acknowledgments}

We are grateful to Mathis Plapp (Ecole Polytechnique, Palaiseau) for numerous fruitful discussions. One of the authors (M.S.) was supported by the Centre National d'Etudes Spatiales (CNES), France.

\section{References}

[1] Hunt JD, Jackson KA. Trans Metall Soc AIME 1966;236:1129.

[2] Jackson KA, Hunt JD. Trans Metall Soc AIME 1966;236:843.

[3] Karma A, Sarkissian A. Met. Trans. A 1996; 27:635.

[4] Ginibre M, Akamatsu S, and Faivre G. Phys Rev E 1997;56:780.

[5] Akamatsu S, Plapp M, Faivre G, Karma A. Metall Mat Trans A 2004;35:1815. 
[6] Kraft RW. Trans. Metall Soc. AIME 1962;224:65

[7] Hecht U, Witusiewicz VT, Drevermann A, Rex S. Trans. Indian Inst. Met. $2005 ; 58: 545$

[8] Hogan LM, Kraft RW, Lemkey FD. Adv. Mater. Res. 1971;5:8.

[9] Akamatsu S, Moulinet S, Faivre G. Metall Mater Trans A 2001;32:2039.

[10] Caroli B, Caroli C, Faivre G, Mergy J. J Cryst Growth 1992;118:135.

[11] Favier JJ, Turpin M, Petitpas C, Labulle B. J Cryst Growth 1977;38:109.

[12] Akamatsu S, Bottin-Rousseau S, Perrut M, Faivre G, Sturz L, Witusiewicz VT. J Cryst Growth 2007;299:418.

[13] Faivre G. In: Rappaz M, Beckermann C, Trivedi R, editors. Solidification Processes and Microstructures : A Symposium in Honor of Prof. W. Kurz. Warrendale PA: The Minerals, Metal and Materials Society 2004. p. 239-244

[14] Sutton AP, Balluffi R.W. Interfaces in Crystalline Materials Oxford University Press; 2009.

[15] Hoffmann DW, Cahn J.W. Surf Science 1972; 31:368.

[16] Akamatsu S, Faivre G, Ihle T. Phys Rev E 1995;51:4751.

[17] Herring C. Phys Rev 1951;82:87.

[18] Börzsönyi T, Akamatsu S, Faivre G. Phys Rev E 2009;80:051601.

[19] Debierre JM, Karma A, Célestini F, Guérin R. Phys Rev E 2003;68:041604.

[20] Datye W, Langer J.S. Phys Rev B 1981; 24:4155.

[21] Akamatsu S, Bottin-Rousseau S, Faivre G. Acta Mater 2011;59:7586.

[22] Akamatsu S, Bottin-Rousseau S, Faivre G. Phys Rev Lett 2004;93:175701. 


\section{List of figure captions}

Figure 1: Repeat units of lamellar eutectic patterns. a) Isotropic system. b) System with a strong interfacial anisotropy of the interphase boundary in the symmetric-pattern approximation. $a, b$ : eutectic phases. $L$ : liquid. $\mathbf{z}$ : growth direction parallel to the thermal gradient. $\mathbf{y}$ : normal to the sample plane. $\mathbf{x}$ : average direction of the solid-liquid interface. $\phi_{s t}:$ steady-state inclination angle. Other symbols: see the text.

Figure 2: Wulff construction for an interphase boundary. WF: Wulff form. $\hat{\boldsymbol{t}}$ and $\hat{\boldsymbol{n}}$ : unit tangent and normal vectors to the interphase boundary, respectively. See the text for the other symbols.

Figure 3: Sketches of experimentally observed travelling lamellar eutectic patterns. a) Anisotropydriven travelling patterns at $\lambda<\lambda_{\text {tilt }}$. b) Tilted travelling patterns at $\lambda>\lambda_{\text {tilt }}$ in isotropic systems $[3,4]$. 
Figure 4: Properties of a shallow ( $\epsilon=0.01$ ) cusp as described by Eq. 6. (a) Blow-up of the vicinal regions of the Wulff plot (thin line) and Wulff form (thick line) in suitable surface energy and length units. For clarity the plot has been greatly elongated in the $z$ direction. (b) Inclination angle of lamellae as a function of grain orientation. Points $c$ and $c^{\prime}$ : endpoints of the facet $\left(\phi=0_{ \pm}\right)$. Points $p$ and $p^{\prime}$ : limits of the trough $(\phi= \pm \Phi)$.

Figure 5: Properties of a deep $(\epsilon=0.05)$ cusp as described by Eq. 6. Same symbols as in Fig. 4. Points $e$ and $e^{\prime}$ : edges of equilibrium facets. Dotted lines: unstable orientation ranges of the interphase interface.

Figure 6: Real-time observation of lamellar eutectic patterns during standard thin-sample directional solidification of the $\mathrm{CBr}_{4}-\mathrm{C}_{2} \mathrm{Cl}_{6}$ eutectic. Growth velocity: $0.5 \mu \mathrm{ms}^{-1}$. Sample thickness: $12 \mu \mathrm{m}$. The $\mathbf{z}$ axis is directed vertically upward. The melt is visible above the solid. The different micrographs correspond to different EGs and show the following types of lamellar patterns: a) 2D isotropic; b) 2D locked; c) out-of-plane. The angle of the lamellar plane and $\mathbf{y}$ in c) is $10 \pm 3^{\circ}$. Bar: $20 \mu \mathrm{m}$.

Figure 7: a) RDS experiment (sketch). A thin sample placed on a cold $\left(\mathrm{T}_{-}\right)$and a hot $\left(\mathrm{T}_{+}\right)$ oven is rotated about the $y$ axis. $\Omega$ : centre of rotation. $\theta$ : angle of rotation $(\theta>0$ refers to a clockwise rotation of the sample.) xz: laboratory reference frame. $\mathbf{X Z}$ : sample reference frame. Arrows: directions of motion of the solid-liquid interface in the sample reference frame. RDS lamellar trajectories have been schematically represented as semi-circles. b) Enlarged view of the solidification front. O: zero-growth rate point. M: instantaneous position of a trijunction. $R, \rho$ : coordinates of $\mathrm{M}$ in the laboratory reference frame. 